\title{
APRENDIZAGEM MOTORA EM CRIANÇAS COM PARALISIA CEREBRAL
}

\author{
MOTOR LEARNING IN CHILDREN WITH \\ CEREBRAL PALSY
}

\author{
Carlos Bandeira de Mello Monteiro ${ }^{1}$ \\ Cristiane Matsumoto Jakabi ${ }^{2}$ \\ Gisele Carla dos Santos Palma ${ }^{2}$ \\ Camila Torriani-Pasin ${ }^{3}$ \\ Cassio de Miranda Meira Junior ${ }^{1}$
}

Monteiro C. B. M. et al. Aprendizagem motora em crianças com paralisia cerebral. Rev. Bras. Cresc. e Desenv. Hum. 2010; 20(2): 250-262.

\section{Resumo}

Introdução: a Paralisia Cerebral (PC) tem como característica causar alterações na postura e movimento que dificultam a realização de atividades funcionais. Diante das dificuldades motoras, a reabilitação torna-se essencial e tem como uma opção basear-se na aprendizagem motora. Porém, é importante a investigação do processo de aprendizagem motora em indivíduos com PC para viabilizar a organização de programas de tratamento mais efetivos. Objetivo: analisar o processo de aprendizagem motora em crianças com PC. Método: Para a realização deste trabalho utilizou-se um grupo experimental (GE) e um grupo controle (GC) ambos formados por 4 crianças pareadas em relação ao gênero (um do gênero feminino e três do gênero masculino) e idade (entre sete e doze anos). A tarefa consistia em realizar um caminho em um labirinto, no menor tempo possível. O trabalho consistiu de duas fases, sendo inicialmente a fase de aquisição (AQ) e depois as transferências (Imediata-TI; Curto Prazo-TC e Longo Prazo-TL). Resultado: Verificou-se que não houve diferença estatisticamente significante entre a $\mathrm{AQ}$ e as transferências avaliadas com os valores a seguir: TI $(z=-1,83$ e $p=0,07)$, TC $(z=-1,83$ e $p=0,07)$ e a TL [GE $(z=-1,83$ e $p=0,07)$ e GC $(z=-1,46$ e p $=0,14)]$. Conclusão: No processo de aprendizagem da tarefa de labirinto, analisando-se os resultados entre as fases de AQ e Transferência não se observou diferença, ou seja, os indivíduos com PC mostraram capacidade de aprendizagem preservada por meio da adaptação da tarefa, fato este que ocorreu de forma equivalente aos indivíduos sem paralisia cerebral.

Palavras-chave: paralisia cerebral; aprendizagem em labirinto; criança.

1 Doutores pela Universidade de São Paulo e Professores do curso de Ciências da Atividade Física da Universidade de São Paulo (EACH-USP).

2 Graduadas em Fisioterapia pelo Centro Universitário das Faculdades Metropolitanas Unidas (FMU).

3 Doutora pela Universidade de São Paulo e professora na Escola de Educação Física e Esporte da Universidade de São Paulo (EEFE-USP).

Trabalho realizado no departamento de Fisioterapia do Centro Universitário das Faculdades Metropolitanas Unidas.

Autor responsável pela correspondência: Prof. Dr. Carlos Bandeira de Mello Monteiro. Rua Fidêncio Ramos 128 apt 22; cep 04551-010; cel (11) 99530716; e-mail: carlosfisi@uol.com.br 


\begin{abstract}
Introduction: cerebral palsy (CP) has the characteristic of causing changes in posture and movement that hamper the achievement of functional activities. In the face of motor disabilities, rehabilitation becomes essential and is an option based on motor learning. However, it is important to research the motor learning process in individuals with CP to make the organization of treatment programs more effective. OBJECTIVE: Analyse the motor learning in children with CP. METHOD: For the realization of this work, an experimental group (EG) and a control group (CG) was used, comprised of four children matched in relation to gender (one female and three masculine) and age (between seven and twelve years). The task was to conduct a path into a maze in the shortest time possible. The work consisted of two phases, being initially an acquisition phase (AQ) and then transfers (immediate-IM; short-term-ST and long-term-LT). RESULT: It was found that there was no statistical significance difference between AQ and transfers evaluated with the following values: IM $(z=-1.83, p=0.07), S T(z=-1.83, p=0.07)$ and LT [EG $(z=-1.83$, $\mathrm{p}=0.07)$ and $\mathrm{CG}(\mathrm{z}=-1.46, \mathrm{p}=0.14)]$. CONCLUSION: In the process of maze task learning, when analyzing the results between phases AQ and transfer, significant difference was not observed, which means that individuals with CP showed learning capacity through task adaptation equivalent to individuals without cerevral palsy.
\end{abstract}

Key words: cerebral palsy; maze learning; children.

\section{INTRODUÇÃO}

A Paralisia Cerebral (PC) pode ser definida como uma desordem da postura e do movimento, persistente, porém não imutável causada por lesão no Sistema Nervoso Central (SNC) em desenvolvimento, antes, durante o nascimento ou nos primeiros meses da infân$\mathrm{cia}^{1,2}$. Stokes ${ }^{2}$ cita que os distúrbios de postura e movimento podem ser definidos como a falta de capacidade do corpo em enfrentar com eficiência os efeitos da gravidade e de relacionar-se com a superfície da terra por meio da base de apoio. Desta forma, a PC causa dificuldades variáveis na coordenação da ação muscular, com resultante incapacidade da criança em manter posturas e realizar movimentos normais ${ }^{1,3-7}$. Neste sentido, os indivíduos apresentam alterações motoras complexas sendo que os déficits primários descritos por Papavasiliou ${ }^{8}$ são: tônus muscular anormal influenciando a postura e movimento; alteração do balance e coordenação; diminuição de força; perda do controle motor seletivo com problemas secundários de contraturas e deformidades ósseas.
Considerando-se as alterações apresentadas e a dificuldade em realizar alinhamento e retificação de posturas que permitam vivenciar suas atividades diárias, é fundamental para o indivíduo com PC a inclusão em programas de habilitação e reabilitação contínuos, os quais podem interferir de forma significativa na interação da criança em contextos relevantes. Deste modo, é possível influenciar no desempenho não só de marcos motores básicos (rolar, sentar, engatinhar e andar), mas também de atividades da rotina diária, como tomar banho, alimentar-se, vestir-se, locomover-se em ambientes variados, entre outras. Devido à insuficiência de respostas sobre benefícios de programa de reabilitação na melhora do indivíduo com $\mathrm{PC}^{8} \mathrm{e}$ ao aumento de interesse pela função motora ${ }^{9,10}$, o fisioterapeuta e outros profissionais da saúde que trabalham com PC podem aplicar os conhecimentos advindos da aprendizagem motora para organizar um programa de tratamento baseado em evidências científicas provenientes desta área básica de conhecimento ${ }^{8}$.

Como fenômeno, a aprendizagem motora pode ser definida como a capacidade do 
indivíduo em desempenhar uma habilidade motora induzindo uma melhora relativamente permanente no desempenho, devido à prática ou à experiência ${ }^{11,12}$. Em outras palavras, é o processo ao longo do qual as habilidades tornam-se facilmente desempenhadas com auxílio de prática e de informação ${ }^{13-15}$. Durante a prática de aquisição da habilidade, o aprendiz necessita executar tentativas para alcançar um desempenho critério ou estabilizar um comportamento ${ }^{11,13}$.

Ao longo desse processo, ocorre a seleção entre os sistemas de memória que permitem a aquisição de alguns aspectos mais relevantes para a cognição, emoção e atenção ${ }^{16}$, bem como a aquisição de estruturas cognitivas, tais como programas ou planos de ação ${ }^{11}$, esquemas $^{17}$ ou $\operatorname{traços}^{18}$, ou a formação de sinergias neuromusculares complexas ${ }^{19}$, dependendo de qual arcabouço teórico é escolhido para dar suporte aos conhecimentos relativos à aprendizagem.

Retenção e transferência são outros dois conceitos cruciais no processo de aprendizagem motora ${ }^{13,17,20}$. São formas de observação e testagem da aprendizagem (vista como fenômeno) que medem, respectivamente: o grau de permanência do que foi adquirido após um período sem prática (retenção) e a capacidade de adequação de um comportamento motor praticado em um contexto diferente, mediante alteração na tarefa motora (transferência).

Assim, para verificar se a aprendizagem ocorreu com solidez, mais do que comparar o desempenho na fase inicial em relação à fase final de aquisição, é necessário recorrer ao desempenho em testes de aprendizagem, isto é, de retenção e/ou de transferência ${ }^{21-24}$. Diante do exposto, é fundamental que o profissional que atua com o indivíduo com PC utilize instrumentos de fácil aplicação e que permitam analisar os fatores que interferem nas fases de aquisição, retenção e transferência de habilidades motoras $^{25}$. Uma tarefa que permite avaliar diversos aspectos neuropsicológicos de planeja- mento, execução, organização espacial e memória implícita e que envolve a operacionalização da intenção de movimentar-se para alcançar um objetivo - e o planejamento desta ação - é a tarefa de labirinto. Esta consiste em realizar no computador um caminho, no menor tempo possível, em um labirinto com uma entrada e uma saída e um único caminho a ser percorrido. Sendo assim, a estabilização do desempenho pode indicar a utilização de estratégias cognitivas e formação de um programa de ação, os quais poderão ser testados na transferência ${ }^{25}$.

Souza et al (2006) ${ }^{25}$ afirmam que a tarefa do labirinto pode ser aplicada na avaliação diagnóstica de indivíduos com alterações no controle e na aprendizagem motora, a fim de identificar que aspectos estão comprometidos durante a execução de uma tarefa motora: processamento da informação e planejamento estratégico (número de erros), função executiva (tempo de execução da tarefa), aprendizagem (estabilização do desempenho) e memória espacial (manutenção do desempenho após tarefa distratora e intervalo de retenção). Além disso, tarefas de labirintos têm a vantagem de poderem ser adaptadas para um número diverso de sujeitos, na medida em que requerem habilidades motoras básicas, podendo ser aplicadas em crianças, idosos e pessoas acometidas por alterações neurológicas ${ }^{25}$.

Assim, o objetivo é verificar o processo de aprendizagem motora da tarefa de labirinto de crianças com PC em comparação a crianças sem alterações da postura e movimento, da mesma faixa etária. O conhecimento mais aprofundado acerca do processo de aprendizagem de uma habilidade motora em indivíduos com PC pode contribuir significativamente para a organização adequada e efetiva de programas de tratamento para essa população.

\section{MÉTODO}

Este estudo foi aprovado pela Comissão de Ética para análise de Projetos de Pesquisa 
da Universidade Cidade de São Paulo sob protocolo CEP número 13364889 e desenvolvido mediante assinatura de um termo de consentimento livre e esclarecido assinado pelo (a) responsável legal dos participantes.

O grupo experimental (GE) foi formado por 4 (quatro) indivíduos com PC residentes na região metropolitana de São Paulo e que frequentavam a Clínica de Fisioterapia do Centro Universitário das Faculdades Metropolitanas Unidas (FMU). O grupo controle (GC) foi formado por 4 (quatro) crianças sem alterações da postura e movimento. Os grupos foram pareados em relação a gênero (um do gênero feminino e três do gênero masculino) e idade (entre sete e doze anos).

Os critérios de inclusão para a participação do estudo foram: aceite de participação no trabalho por meio da assinatura do termo de consentimento livre e esclarecido realizado por um dos responsáveis pelo paciente, diagnóstico médico de PC e alterações motoras que caracterizam indivíduos com diparesia espástica (alterações motoras mais evidentes em membros inferiores). Somente participaram desta pesquisa indivíduos com nível II, segundo o Gross Motor Function Classification System (GMFCS), desenvolvido por Palisano et al. $(1997)^{26}$. Este sistema classifica crianças com PC em cinco níveis de acordo com a função motora, o que significa que todos os pacientes avaliados tinham condições de andar sem dispositivos auxiliadores da mobilidade.

Para a caracterização dos indivíduos e viabilização de um grupo homogêneo utilizouse, também, a Classificação Internacional de Funcionalidade Incapacidade e Saúde (CIF) ${ }^{27}$. Gunnar e Stucki (2007) ${ }^{28}$ afirmam que a CIF é um instrumento que pode ser utilizado para caracterização de grupos em pesquisas científicas. Segundo a Organização Mundial da Saúde (OMS), trata-se de um sistema de classificação útil para a organização de grupos homogêneos em pesquisas científicas, servin- do como uma linguagem para a classificação da funcionalidade e capacidade. Diante disso, os indivíduos selecionados para este trabalho apresentavam a mesma característica funcional no domínio de "funções do corpo" (funções neuromusculo-esqueléticas, relacionadas ao movimento e funções mentais) e no domínio de "atividades e participação" (mobilidade, realização de tarefas, comunicação, habilidades básicas, concentração e atenção). Dessa forma, considerando-se as funções do corpo, os indivíduos avaliados apresentavam funções músculo-esqueléticas relacionadas ao tônus muscular e ao controle dos movimentos voluntários com deficiência leve (respectivamente b735.1 e b760.1) e funções mentais de orientação, intelectuais e de atenção também com deficiência leve (respectivamente b114.1, b117.1 e b140.1).

Considerando-se atividade e participação, quanto à mobilidade, os indivíduos conseguem manter-se sentado sem dificuldades (d4153.0) e apresentam dificuldade leve para pegar e manipular objetos (respectivamente: d4400.1 e d4402.1). Quanto à tarefa e demanda, não têm dificuldade para realizar uma tarefa complexa (d2101.0). No que diz respeito à comunicação, não apresentam dificuldades na recepção, produção e conversação (respectivamente: d310.0, d330.0 e d350.0). Quanto à aprendizagem e aplicação de conhecimentos, não apresentam dificuldades em habilidades básicas (d1550.0) e apresentam dificuldade leve em concentração/atenção e habilidades complexas (respectivamente: d160.1 e d1551.1).

Os critérios de exclusão foram: presença de deformidades ósteo-articulares estruturadas e realização de cirurgia ou bloqueio químico neuromuscular há menos de 6 (seis) meses em membros superiores; outras doenças associadas e indivíduos com alterações nas funções cognitivas que impedissem a colaboração e compreensão de ordens simples nas atividades propostas. 


\section{Instrumentação e delineamento}

Utilizou-se como instrumento de avaliação a tarefa (ou paradigma) de labirinto que foi utilizada pela sua facilidade e adaptabilidade para analisar a aprendizagem de uma habilidade motora ${ }^{25}$. Para realização da tarefa, optou-se pelo programa desenvolvido pelo Departamento de Matemática da Universidade Federal do Rio Grande do Sul, a apresentado por Souza et al. (2006),

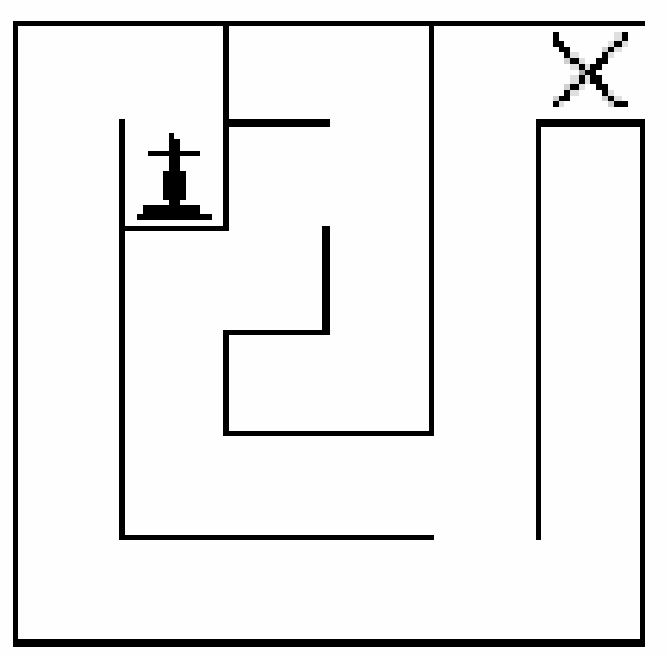

Figura 1: Desenho de labirinto da fase de aquisição.

Fonte: Sousa et al. (2006) ${ }^{25}$

O experimento consistiu de quatro fases (tabela 1). Primeiro, realizou-se o pré-teste, composto de três tentativas a fim de possibilitar ao participante o conhecimento da tarefa. Na segunda fase (Aquisição - AQ), os indivíduos praticaram 30 tentativas ao longo das quais o sujeito completou o percurso da figura 1. A terceira fase foi a TI, que consistiu em, após 5 minutos de descanso, realizar 5 tentativas utilizando o labirinto da figura 2. Na quarta fase (TC), os sujeitos repetiram a tarefa da TI depois de mais 5 minutos de descanso. A quinta e última fase foi a TL, realizada sete disponibilizado no site www.mat.ufrgs.br. Dois desenhos de labirintos com apenas um caminho correto a ser percorrido foram escolhidos; o primeiro foi utilizado na fase de aquisição (AQ) e o segundo nas fases de transferência (Transferência imediata - TI, Transferência de curto prazo - TC e Transferência de longo prazo - TL). As figuras 1 e 2 ilustram as variações da tarefa em cada fase do experimento.

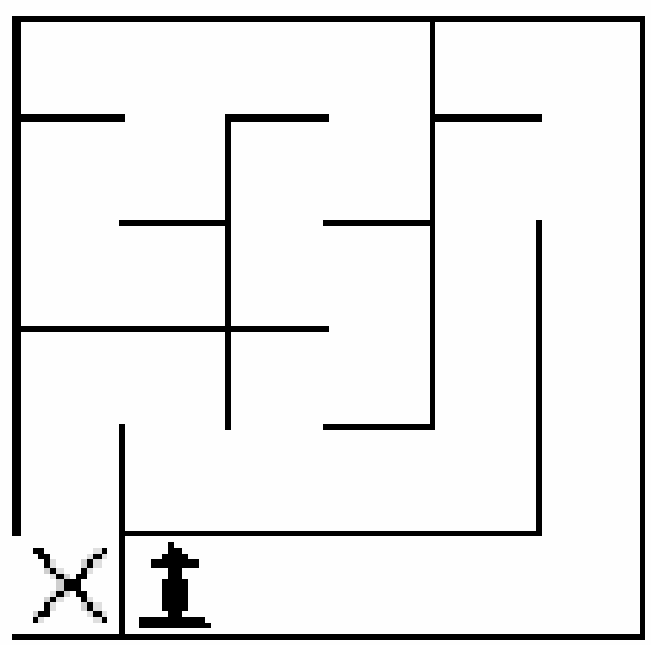

Figura 2: Desenho de labirinto das fases de transferência.

Fonte: Sousa et al. (2006) $)^{25}$

dias depois, na qual os indivíduos executaram a mesma tarefa da TI e da TC.

\section{Procedimentos}

As crianças foram recrutadas individualmente em sala apropriada com um computador, mesa, cadeira e a participação de um avaliador responsável pela instrução e anotação dos valores de tempo em papel, com tabela dos números das tentativas. Os valores foram marcados considerando-se o tempo total da finalização da tarefa, valor fornecido pelo próprio programa. Optou-se em utilizar a tarefa 
Tabela 1: Tabela do delineamento experimental

\begin{tabular}{|c|c|c|c|c|c|c|}
\hline & Pré-teste & & Aquisição & TI & TC & TL \\
\hline & 3 tentativas & & 30 tentativas & 5 tentativas & 5 tentativas & 5 tentativas \\
\hline GE & BL1 & BL2 & $\begin{array}{l}\text { BL3 BL4 BL5 BL6 } \\
\text { Labirinto } 1\end{array}$ & $\begin{array}{c}\text { BL1 } \\
\text { Labirinto } 2 \\
\end{array}$ & $\begin{array}{c}\text { BL1 } \\
\text { Labirinto } 2 \\
\end{array}$ & $\begin{array}{c}\text { BL1 } \\
\text { Labirinto } 2 \\
\end{array}$ \\
\hline GC & $\begin{array}{c}3 \text { tentativas } \\
\text { BL1 }\end{array}$ & BL2 & $\begin{array}{l}30 \text { tentativas } \\
\text { BL3 BL4 BL5 BL6 } \\
\text { Labirinto } 1\end{array}$ & $\begin{array}{l}5 \text { tentativas } \\
\text { BL1 } \\
\text { Labirinto } 2\end{array}$ & $\begin{array}{l}5 \text { tentativas } \\
\text { BL1 } \\
\text { Labirinto } 2\end{array}$ & $\begin{array}{l}5 \text { tentativas } \\
\text { BL1 } \\
\text { Labirinto } 2\end{array}$ \\
\hline
\end{tabular}

GE: Grupo experimental; GC: Grupo controle; BL: Bloco; Labirinto 1: fase de aquisição (AQ); Labirinto 2: fases de transferência; (TI) Transferência Imediata; TC Transferência de curto prazo; (TL) transferência de longo prazo

de labirinto no computador, por ser um instrumento tecnológico e facilitador para indivíduos com $\mathrm{PC}^{29}$.

Cada criança foi posicionada adequadamente, sentada à frente da tela do computador; a tarefa foi explicada concomitantemente com a apresentação do labirinto, ao longo do qual a criança deveria percorrer o caminho com o desenho (apontado na tela pelo avaliador) até a saída do labirinto identificado por um " $\mathrm{X}$ " (apontado na tela pelo avaliador). A criança foi instruída a executar a tarefa o mais rápido possível utilizando os botões do teclado identificados pelas setas de: acima, abaixo, lateral direita e lateral esquerda.

Os dados foram organizados em blocos (BL) de seis tentativas e analisados por meio de análises descritivas e inferenciais.

\section{Análise dos dados}

Em virtude da quantidade reduzida de observações e pela violação dos pressupostos de normalidade e igualdade de variâncias, os dados foram analisados por meio de técnicas não paramétricas. Três análises foram desenvolvidas:

1) Intragrupo de aquisição: com vista a detectar a evolução do desempenho do início para o final da aquisição, comparou-se os blocos de aquisição em cada um dos grupos separadamente, por intermédio da análise de variância de Friedman.
2) Intragrupo de transferência: comparação em cada grupo separadamente entre o último bloco de aquisição e cada uma das fases de transferência (teste de Wilcoxon).

3) Intergrupo de transferência: comparação em cada uma das três fases separadamente do desempenho do GC com o do GE (teste U de Mann-Whitney).

Para todos os testes, o nível de significância estatístico adotado foi de 5\%. O programa estatístico foi o SPSS (Statistical Package for Social Sciences), versão 13.0.

\section{RESULTADOS}

A seguir, os resultados serão apresentados em duas partes: caracterização da amostra e estatística dos dados.

\section{a) Caracterização da amostra}

A seguir, estão as características dos indivíduos do GE com a idade, gênero, valores do GMFCS e CIF (tabela 2).

\section{b) Estatística dos dados}

A tabela 3 mostra os resultados, em segundos, da tarefa de labirinto do GE e do GC com os valores dos seis blocos da fase de aquisição, seguidos dos valores das transferências. 
Tabela 2: Caracterização da amostra do grupo experimental (GE).

\begin{tabular}{|c|c|c|c|c|}
\hline Sujeito & Idade & Gênero & GMFCS* & $\mathrm{CIF}^{* *}$ \\
\hline 1 & 7 anos & Masculino & & Funções do corpo \\
\hline 2 & 8 anos & Masculino & & $\begin{array}{c}\text { b114.1; b117.1; b140.1; b735.1 } \\
\text { e b760.1 }\end{array}$ \\
\hline 3 & 11 anos & Masculino & II & Atividades e Participação \\
\hline 4 & 12 anos & Feminino & & $\begin{array}{c}\mathrm{d} 4153.0 ; \mathrm{d} 4400.1 ; \mathrm{d} 4402.1 ; \\
\mathrm{d} 2101.0 ; \mathrm{d} 310.0 ; \mathrm{d} 330.0 \\
\mathrm{~d} 350.0 ; \mathrm{d} 1550.0 ; \mathrm{d} 160.1 \\
\text { e d1551.1 }\end{array}$ \\
\hline
\end{tabular}

*GMFCS (Gross Motor Function Classification System); **CIF (Classificação Internacional de funcionalidade, incapacidade e saúde); II- segundo nível do Sistema de Classificação da Função motora Grossa.

Tabela 3: Resultados (segundos) dos blocos de aquisição e das transferências.

\begin{tabular}{ccccccccccccc}
\hline INDIVÍDUOS & GRUPO & IDADE & GÊNERO & BL1 & BL2 & BL3 & BL4 & BL5 & BL6 & TI & TC & TL \\
\hline 1 & GE & 7 & M & 133 & 70 & 70 & 69 & 70 & 68 & 100 & 98 & 178 \\
2 & & 8 & M & 94 & 81 & 69 & 66 & 64 & 53 & 109 & 60 & 63 \\
3 & & 11 & M & 25 & 19 & 18 & 23 & 15 & 18 & 23 & 20 & 21 \\
4 & & 12 & F & 26 & 30 & 27 & 28 & 22 & 21 & 36 & 31 & 27 \\
\hline 5 & GC & 7 & M & 57 & 41 & 40 & 48 & 43 & 36 & 67 & 60 & 49 \\
6 & & 8 & M & 63 & 50 & 33 & 35 & 38 & 40 & 56 & 57 & 49 \\
7 & & 11 & M & 72 & 49 & 49 & 35 & 35 & 34 & 63 & 38 & 32 \\
8 & 12 & F & 42 & 23 & 25 & 26 & 23 & 21 & 63 & 30 & 29 \\
\hline
\end{tabular}

GE- Grupo Experimental; GC- grupo controle; BL- bloco; TI- Transferência Imediata;TCTransferência a curto prazo; TL- Transferência a longo prazo.

\section{b.1) Análise intragrupo de aquisição}

O objetivo dessa análise foi identificar em cada um dos grupos a existência de melhora de desempenho ao longo dos seis blocos de aquisição. A Anova de Friedman demonstrou diferenças estatisticamente significativas tanto no GE $\left(X^{2}=12,78 ; p=0,026\right)$ como no GC $\left(X^{2}=11,72 ; \mathrm{p}=0,039\right)$. A direção das diferenças pode ser identificada com a inspeção das medianas (tabela 4), que indica, em ambos os grupos, a queda do tempo para realizar a tarefa. Portanto, pode-se inferir que houve melhora significativa do desempenho do início para o final na fase de aquisição inclusive para o grupo com Paralisia Cerebral.

\section{b.2) Análise intragrupo de transferência}

As comparações entre o último bloco da aquisição com cada bloco da transferência - TI, TC e TL - foram realizadas por intermédio de três análises pareadas (BL6 X TI, BL6 X TC e BL6 X TL) de Wilcoxon. Verificou-se que não houve diferença significativa entre o BL6 e a TI para ambos os grupos $(\mathrm{z}=-1,83$ e $\mathrm{p}=0,07)$, entre o BL6 e a TC para ambos os grupos ( $\mathrm{z}=-1,83$ e $\mathrm{p}=0,07)$ e entre o BL6 e a TL [GE $(\mathrm{z}=-1,83$ e $\mathrm{p}=0,07)$ e $\mathrm{GC}(\mathrm{z}=-1,46 \mathrm{e} \mathrm{p}=0,14)]$. Assim, por 
Tabela 4: Valores das medianas (em segundos) em cada grupo nos seis blocos de aquisição.

\begin{tabular}{ccc}
\hline Blocos & GE & GC \\
\hline 1 & 5,25 & 6 \\
2 & 4,75 & 3,75 \\
3 & 3,63 & 2,88 \\
4 & 3,75 & 3,63 \\
5 & 2,25 & 3 \\
6 & 1,38 & 1,75 \\
\hline
\end{tabular}

GE - grupo experimental; GC- grupo controle

não ocorrer diferença significativa, pode-se inferir que ocorreu adaptação à modificação da tarefa. Isso pode ser interpretado como refletindo que os sujeitos adquiriram a capacidade de transferir o que foi praticado na aquisição para uma situação nova, imposta pela modificação da tarefa de labirinto.

\section{b.3) Análise intergrupo de transferência}

Os testes U de Mann-Whitney, que compararam o GE e o GC em cada uma das transferências, não indicaram diferenças significativas entre os grupos na TI ( $\mathrm{z}=0$ e p $=1)$, na TC $(z=-0,15 ; p=0,88)$ e na TL $(z=0$ e $p=1)$.

\section{DISCUSSÃO}

A pergunta de pesquisa que se apresenta neste estudo é: indivíduos com PC - que possuem alterações na postura e na capacidade de movimentação - conseguem aprender uma tarefa motora e realizá-la de forma funcional, com capacidade para ajustar as novas demandas ambientais? A tarefa escolhida foi a de labirinto, que implica em movimentação motora fina para manuseio do teclado do computador por meio de segmentos corporais superiores; portanto, pode ser considerada uma ação motora cotidiana de grande importância nos dias atuais ${ }^{29}$. Na tarefa de labirinto, crianças sem alterações motoras têm como principal desafio escolher o caminho correto; já os indivíduos com PC têm, além da escolha do caminho correto, dificuldade em realizar o movimento.

De acordo com os resultados obtidos, verificou-se que toda a amostra (GE e GC) apresentou diminuição significativa do tempo de execução da tarefa entre o primeiro e o último BL da aquisição. Isso significa melhora de desempenho ocorrida em função da prática, o que permite analisar os testes de transferência e, a partir deles, inferir ocorrência de aprendizagem. Durante o processo, o aprendiz passa de uma fase inicial - caracterizada por elevado número de erros, inconsistência e alta demanda de atenção - para uma fase posterior, que se caracteriza por consistência, poucos erros e demanda reduzida de atenção. Com a prática, ocorrem menos movimentos desnecessários e consequente otimização de energia e diminuição no tempo de realização da tarefa, fazendo com que a sequência de movimentos ganhe progressivamente fluência e harmonia. Segundo definições clássicas de habilidade motora, a prática com informação complexa e intencional envolve mecanismos (perceptivo, decisório e efetor) que, mediante o processo de aprendizagem, torna-se organizada e coordenada de tal forma a alcançar objetivos predeterminados com máxima certeza e mínimo dispêndio de energia ${ }^{30,31}$.

Assim, a prática proporciona a estabilização funcional na tarefa, ou o alcance de controle ótimo na execução, teoricamente levando à padronização funcional, momento a partir do qual o sistema está apto a adaptar-se a novas condições ${ }^{11,32}$. A padronização da função é alcançada por meio de prática e feedback negativo. Deste modo, o aprendiz passa por um processo de diferenciação, em que um estado instável, geral e homogêneo dá lugar a um estado estável, específico e heterogêneo ${ }^{33}$. Nas tentativas iniciais, a magnitude e variabilidade dos erros são grandes, caracterizando os movimentos como inconsistentes e desordenados. Com as repetições, a relação meio-fim para 
atingir a meta da tarefa é reforçada até que se alcancem estados estáveis, ou seja, a execução de movimentos torna-se ordenada e consistente, caracterizada pela redução da magnitude e variabilidade dos erros.

Vale ressaltar que, pela comparação descritiva dos valores das medianas na aquisição, o desempenho dos indivíduos com PC foi melhor que dos indivíduos sem PC, na maioria dos blocos. Esse resultado na aquisição pode ser discutido, provavelmente, pela motivação durante a execução da tarefa; os indivíduos com PC mostraram-se mais motivados, ao passo que os indivíduos sem PC parecem ter considerado a tarefa fácil e desmotivadora. A motivação é um dos fatores que afetam o processo de aprendizagem motora, sobretudo na fase de aquisição porque viabiliza um melhor processamento de informações, favorecendo a retenção e a transferência do que foi adquirido ao longo do processo de prática ${ }^{11,13,32}$. Ainda neste âmbito, a motivação parece afetar também o desempenho de habilidades motoras, uma vez que faz o aprendiz prestar mais atenção na execução da tarefa, aumentando o envolvimento e a participação ${ }^{34-36}$.

Os resultados mais importantes do presente estudo dizem respeito às comparações intra e entre os grupos na fase de transferência, já que a modificação da tarefa exige dos aprendizes a capacidade de adaptação. O objetivo precípuo da prática não é facilitar a performance de efeitos temporários durante a aquisição, mas sim possibilitar melhor desempenho duradouro (atribuído à aprendizagem) nos testes de retenção e transferência ${ }^{11,13,37,38}$. Então, um aspecto fundamental da aprendizagem motora é que as habilidades são executadas em ambientes que demandam constante capacidade de adaptação ${ }^{11,13}$. Esta adaptação, no entanto, só ocorre na medida em que existam situações desafiadoras à capacidade de movimento já adquirida, de forma que novas estruturas de ação tenham que ser formadas para atender às exigências impostas pelo ambiente. Para tan- to, inicialmente o indivíduo precisa identificar o problema motor que, a título de exemplo, poderíamos colocar a tarefa de labirinto.

Nos testes de labirinto têm-se a possibilidade de avaliar diversos aspectos neuropsicológicos, tais como, função executiva, aprendizagem espacial e memória implícita ${ }^{25}$. Após a identificação do problema, o sujeito precisa formular um plano de ação, em que deverá gerar uma hipótese de como seria possível realizar tal objetivo, prevendo e antecipando-se frente à execução da ação. $O$ passo seguinte consiste em colocar em prática o plano de ação estabelecido, transformando uma imagem mental em movimentos efetivos. Nessa fase, é preciso coordenar um complexo sistema muscular, a fim de que vários músculos sejam ativados de forma organizada. Como raramente a transformação de planos de ação em movimento é perfeita nas tentativas iniciais, o indivíduo precisa analisar os erros cometidos e tentar corrigí-los nas tentativas seguintes. Quando o ciclo descrito acima é repetido suficientemente, ocorre a transição de ações mal-coordenadas de grande demanda cognitiva para movimentos altamente precisos, em que sinergias neuromusculares complexas são ativadas com mínimo envolvimento da atenção. A esse processo dá-se o nome de aprendizagem ${ }^{19}$.

A análise intragrupo do último bloco de aquisição, para cada uma das transferências indicou ausência de diferenças em ambos os grupos. Portanto, uma situação mais complexa, de alteração da tarefa, não ocasionou queda significativa de desempenho. Assim, os sujeitos, com PC e os sujeitos do GC, apresentaram boa adaptabilidade a uma situação nova na tarefa de labirinto. As análises entre os grupos na transferência confirmam a boa capacidade de adaptação de ambos os grupos sem diferenças entre eles, haja vista a ausência de diferença significativa de desempenho (tempo para execução da tarefa) entre o GE e o GC, em todos os testes de transferência.

Portanto, a PC parece não ter tido influência sobre o desempenho da tarefa de labi- 
rinto modificada, fato que se constitui interessante, tanto sob o ponto de vista de caracterização dos sujeitos com PC, quanto sob o ponto de vista da intervenção com esta população. A hipótese inicial de dificuldade para os aprendizes com PC não foi corroborada, talvez porque na fase de aquisição, a motivação e perseverança desses aprendizes tenha sido maior quando comparada aos aprendizes sem PC. Isso pode ter compensado possíveis diferenças na comparação entre os grupos na fase de transferência. Uma das características da aquisição de habilidade motora é a execução da tarefa motora com menor tempo. Os tempos similares entre os grupos na tarefa de labirinto modificada (transferência) demonstram capacidades similares na velocidade de processamento de informações. Muitas tarefas motoras requerem respostas rápidas aos estímulos ambientais, assim como rápidos ajustes ou correções baseados em resultados de desempenhos anteriores. Um fator importante na velocidade de processamento central é a quantidade de informação que envolve os efeitos da complexidade da informação, do tempo utilizável para o processamento e da capacidade do sujeito ${ }^{39,40}$. Parece que tanto os indivíduos com PC, como os sem PC foram capazes de lidar satisfatoriamente com essas três variáveis o que, também, se torna relevante sob o ponto de vista da intervenção com estes sujeitos, seja na reabilitação, seja na prática de atividade física.

Os achados do presente estudo podem ser contrastados com alguns poucos estudos que foram realizados com indivíduos com PC no âmbito da Aprendizagem Motora. O fato de os indivíduos com PC terem aprendido a tarefa corrobora aos achados de Hemayattalab e Rostami (2010) ${ }^{41}$, Reid (2002) ${ }^{42}$ e Chen et al. $(2007)^{43}$ com tarefas de lançamento de dardo e de realidade virtual, nas quais os indivíduos com PC melhoraram a qualidade de movimento. Alguns autores ${ }^{44,45}$ optaram em investigar a aprendizagem de indivíduos com PC por meio de mudanças em estruturas neuronais (via exames de neuroimagem) e verificaram a ocorrência da aprendizagem sob o ponto de vista neural, detectando ativação em áreas corticais e correlações entre a performance na execução de tarefas motoras e a atividade cerebral em diferentes áreas encefálicas.

Pelo fato de haver poucos trabalhos utilizando-se dos conhecimentos advindos da Aprendizagem Motora em indivíduos com PC, é importante enfatizar a necessidade de outros estudos para maior compreensão dos fatores que afetam a aquisição de habilidades motoras nessa população particular. Sugere-se que as investigações futuras em Aprendizagem Motora possam manipular diferentes formas de prática (variabilidade, distribuição, fracionamento) e de informação (feedback extrínseco, demonstração/instrução, estabelecimento de metas, foco interno/externo) ao aprendiz com PC. A variabilidade de prática - que consiste em variações nas características do contexto ou variações da tarefa motora que está sendo praticada - parece ser uma prioridade, haja vista sua implicação direta nas intervenções propostas para estes sujeitos. Em indivíduos com deficiência mental, a prática variada proporcionou aumento da chance de sucesso em novas situações (teste de transferência) ${ }^{46}$; em indivíduos com PC, o papel da variação tem sido apontado como fundamental na geração de estratégias motoras elaboradas ${ }^{47}$.

Desta maneira, no processo de aprendizagem da tarefa de labirinto, tanto os indivíduos com PC como os indivíduos sem PC apresentaram melhora de desempenho, observado por meio da diminuição do tempo de execução ao longo da aquisição. Quanto à aprendizagem, mensurada por meio do teste de transferência, os indivíduos com PC mostraram capacidade de adaptação equivalente aos indivíduos sem PC. 


\section{REFERÊNCIAS}

1. Aicardi J, Bax M. Cerebral palsy. In: Aicardi J. Diseases of the nervous system in childhood. Clinics in developmental medicine. London: Mac Keith Press; 1992. p. 334-932.

2. Stokes M. Neurologia para Fisioterapeutas. São Paulo: Premier; 2000.

3. Parker DF, Carriere L, Hebestreit H, Salsberg A, Bar OR. Muscle Performance and Gross Motor Function of Children With Spastic Cerebral Palsy. Developmental medicine and child neurology. 1993;35(1):17-23.

4. Boyce WF, Gowland C, Rosenbaum PL, Lane M, Plews N, Goldsmith $\mathrm{CH}$, et al. The Gross Motor Performance Measure: Validity and Responsiveness of a Measure of Quality of Movement. Physical therapy. 1995;75(7):603-613.

5. Edwards S. Neurological physiotherapy. New York: Churchill Livingstone; 1996.

6. Bax M. Medical aspects of cerebral palsy. In: Finnie NR. Handling the young child with cerebral palsy at home. 3rd ed. London: Butterworth-Heinemann; 1997.

7. Diament A, Cypel S. Neurologia infantil. 4th ed. São Paulo: Atheneu; 2005.

8. Papavasiliou AS. Management of motor problems in cerebral palsy: A critical update for the clinician. Eur J Paediatr Neurol. 2009; 13(5): 387-396.

9. Bax M. Diagnostic assessment of children with cerebral palsy. Lancet Neurol. 2004;3:395.

10. Cans C. Surveillance of cerebral palsy in Europe: a collaboration of cerebral palsy surveys and registers. Dev Med Child Neurol. 2007;42(12):816-824.

11. Tani G. Aprendizagem motora: tendências, perspectivas e problemas de investigação. In: Tani G. Comportamento motor: aprendizagem e desenvolvimento. Rio de Janeiro: Guanabara Koogan; 2005.
12. Holmefur M, Krumlinde-Sundholm L, Bergstrom J, Eliasson A. Longitudinal development of hand function in children with unilateral cerebral palsy. Developmental Medicine \& Child Neurology. 2009; 52(4): 352-357.

13. Magill R. A. Aprendizagem motora: conceitos e aplicações. 5th ed. São Paulo: Edgard Blücher; 2000.

14. Manoel EJ. A dinâmica do estudo do comportamento motor. Revista Paulista de Educação Física. 1999;13:52-61.

15. Lemieux TS, Penhune VB. The effects of practice and delay on motor skill learning and retention. Experimental Brain Research. 2005;161:423-431.

16. Lent R. Cem bilhões de neurônios: conceitos fundamentais de neurociência. São Paulo:Atheneu; 2001. p. 587-617.

17. Schmidt R, Wrisberg C. Aprendizagem e performance motora: uma abordagem da aprendizagem baseada no problema. 3rd ed. Porto Alegre: Artmed; 2001.

18. Adams JA. A closed-loop theory of motor learning. Journal of Motor Behavior. 1971; 3:111-50.

19. Teixeira LA. Aprendizagem de habilidades motoras na ginástica artística. In: Nunomura M, Nista-Piccolo VL. Compreendendo a ginástica artística. São Paulo: Phorte; 2004.

20. Kleim JA, Jones TA. Principles of experience-dependent neural plasticity: implications for rehabilitation after brain damage. Journal of Speech, Language, and Hearing Research. 2008; 51: 225-239.

21. Mulder TA. A process-oriented model of human motor behavior: toward a theorybased rehabilitation approach. Physical Therapy. 1991; 71: 157-64.

22.7Carr JH, Shepherd RB. The changing face of neurological rehabilitation. Revista Brasileira de Fisioterapia. 2006; 10(2):147-56. 
23. Salmoni A, Schmidt RA, Walter CB. Knowledge of results and motor learning: A review and critical reappraisal. Psychological Bulletin. 1984; 95: 355-386.

24. Emanuel M, Jarus T, Bart, O. Effects of focus of attention and age on motor acquisition, retention, and transfer: a randomized trial. Physical Therapy. 2008; 88(2): 251-60.

25. Souza DE, França FR, Campos TF. Teste de Labirinto: Instrumento de Análise na Aquisição de uma Habilidade Motora. Revista Brasileira de Fisioterapia. 2006; 10(3): 355-60.

26. Palisano R, Rosenbaum P, Walters S, Russel DJ, Wood EP, Galuppi P. Development and reliability of a system to classify gross motor function in children with cerebral palsy. Dev Med Child Neurol. 1997; 39: 214-23.

27. CIF - Classificação Internacional de funcionalidade, Incapacidade e Saúde/ [Centro Colaborador da Organização Mundial da Saúde para Família de Classificações Internacionais, organização e coordenação da tradução Cássia Maria Buchalla]. São Paulo: Editora da Universidade; 2003.

28. Gunnar G, Stucki G. Organizing human functioning and rehabilitation research into distinct scientific fields. Part II: Conceptual descriptions and domains for research. Journal of rehabilitation medicine. 2007;39(4): 293-8.

29. Sullivan KJ, Kantak SS, Burtner PA. Motor Learning in Children: Feedback Effects on Skill Acquisition. Physical Therapy. 2008; 88(6):720-32.

30. Benda RN. Sobre a natureza da aprendizagem motora: mudança e estabilidade... e mudança. Revista Brasileira de Educação Física. 2006; 20(5): 43-45.

31. Pellegrini AM. A aprendizagem de habilidade motoras I: o que muda com a prática? Revista Paulista de Educação Física. 2000; 3: 29-34.
32. Choshi K. Aprendizagem motora como um problema mal-definido. Rev. paul. Educ. Fís. 2000; 3: 16-23.

33. Bertalanffy LV. Teoria geral dos sistemas: aplicação à psicologia. In: Anohin PK, Bertalanffy LV, Rapoport A, Mackenzie WJM, Thompson JD. Teoria dos sistemas. Rio de Janeiro: Fundação Getúlio Vargas; 1976.

34. Paim MCC. Fatores motivacionais e desempenho no futebol. Revista da Educação Física/UEM. 2001; 12(2): 73-79.

35. Valenteini NC. A influência de uma intervenção motora no desempenho motor e na percepção de competência de crianças com atrasos motores. Rev. paul. Educ. Fís. 2002; 16(1); 61-75.

36. Shumway-Cook A, Woollacott MH. Controle motor. Teoria e aplicações práticas. 2nd ed. São Paulo: Manole; 2003.

37. Karni A, Meyer G, Rey-Hipolito C, Jezzard P, Adams MM, Turner R, et al. The acquisition of skilled motor performance: fast and slow experience-driven changes in primary motor cortex. Proceedings of the National Academy of Sciences. 1998; 95: 861-68.

38. Wulf G, Shea G, Lewthwaite R. Motor skill learning and performance: a review of influential factors. Med Educ. 2010; 44: 75-84.

39. Chiviacowsky S, Neves C, Locatelli L, Oliveira C. Aprendizagem motora em crianças: efeitos da freqüência autocontrolada de conhecimento de resultados. Rev. Bras. Cienc. Esporte. 2005; 26(3): 177-190.

40. Chiviacowsky S, Kaefer A, Medeiros FL, Pereira FM. Aprendizagem motora em crianças:"feedback" após boas tentativas melhora a aprendizagem?. Rev. bras. Educ. Fís. Esp. 2007; 21(2): 57-65.

41. Hemayattalab R; Rostami LR. Effects of frequency of feedback on the learning of motor skill in individuals with cerebral 
palsy. Research in Developmental Disabilities. 2010; 31: 212-217.

42. Reid D. The use of virtual reality to improve upper-extremity efficiency skills in children with cerebral palsy: a pilot study. Tech Disabil. 2002;14:53-61.

43. Chen YP, Kang LJ, Chuang TY, Doong JL, Lee SJ, Tsai MW, et al. Use of Virtual Reality to Improve Upper-Extremity Control in Children With Cerebral Palsy: A Single-Subject Design. Physical Therapy. 2007;87(11): 1441-57.

44. Rieckmann A; Bäckman L. Implicit Learning in Aging: Extant Patterns and New Directions. Neuropsychol Rev. 2009;19:490-503.
45. Orban P, Peigneux P, Lungu O, Albouy G, Breton E, Laberenne F, et al. The multifaceted nature of the relationship between performance and brain activity in motor sequence learning. NeuroImage. 2010; 49: 694-702.

46. Hemayattalab R, Movahedi A. Effects of different variations of mental and physical practice on sport skill learning in adolescents with mental retardation. Research in Developmental Disabilities. 2010; 31: 81-86.

47. Straub K, Obrzut JE. Effects of Cerebral Palsy on Neuropsychological Function. J Dev Phys Disabil. 2009; 21:153-167.

Recebido em 28 de março de 2010 Modificado em 16 de abril de 2010 Aceito em 06 de maio de 2010 